\title{
Wavelet Based Energy Extraction Method for Determining Power Quality Disturbances
}

\author{
C. Kocaman ${ }^{1}$, M. Ozdemir ${ }^{2}$ \\ ${ }^{1}$ Faculty of Aeronautics and Astronautics \\ Ondokuz Mayis University \\ Campus of Ballica Samsun (Turkey) \\ Phone/Fax number:+090 36231219 19, e-mail: ckocaman@omu.edu.tr \\ ${ }^{2}$ Department of Electical and Electronics Engineering \\ Ondokuz Mayis University \\ Campus of Kurupelit Samsun (Turkey) \\ Phone/Fax number:+090 36231219 19, e-mail: ozdemirm@omu.edu.tr
}

\begin{abstract}
In this paper, energy of wavelet coefficients are used for determining power quality disturbances (PQD) which are important for power systems. These power quality disturbances are voltage with harmonics, transient and flicker. After analyzing energy coeffients based on wavelet transform, these PQD are compared with healty condition. $50 \mathrm{~Hz}$ pure sine is chosen as reference. These signals are generated by using MATLAB. Sampling frequency is $25.6 \mathrm{kHz}$. Energy distribution of detail coefficients are obtained by using 12 level Daubechies4 discrete wavelet filter. Parseval theorem is applied to wavelet coefficients for obtaining energy distribution of detail coefficients of these relevant disturbances in different resolution levels. Satisfactory results are taken visually when examining energy distrubutions of these PQD. Also it is observed that these PQD can be distinguished visually when their amplitudes are increased. Energy coefficients of PQD and amplitude of PQD are in direct propotion.
\end{abstract}

\section{Key words}

Power quality disturbances, wavelet trasform, harmonics, transients, flicker.

\section{Introduction}

In recent years, researchers focused on power quality problems. Current that have $120^{\circ}$ phase difference and almost same amplitude is wanted to be drawn from three phase source [1]. The quality of the energy is ensured by staying within these criteria. Also, quality of the power can be defined as limitation cluster that allow devices to behave desired manner without the loss of life and performance. Power quality disturbances bring some problems such as instability, short life and malfunction together [2]. Sources and causes of disturbances must be determined before taking the necessary precautions to improve the power quality [3].

The effects of power quality disturbances can be determined and the source of power quality disturbances can be analyzed if disturbances classified correctly [4].

In this paper, pure sine and power quality disturbances such as voltage with harmonics, transient and flicker are dealt with.

Harmonics are voltages or currents that operate at a frequency that is an integer (whole-number) multiple of the fundamental frequency. In a power system, it is desirable that the current and voltage waveforms are sinusoidal. If current and voltage are sinusoidal, there is only basic component, there are not harmonics. The voltage and current harmonic components cause the waveform to deviate from the sinusoidal form. Harmonic components are produced by nonlinear elements with current-voltage characteristics. If sinusoidal voltage is applied to the linear load, the load current does not contain harmonic components and the load current is sinusoidal. Rectifiers, motor drive circuits, power electronic circuits such as inverters, arc furnaces, gas discharge lamps are examples of non-linear loads. Even if the source voltage is sinusoidal, harmonic current will be drawn when a nonlinear load is connected to the network. Therefore, the sinusoidal voltage at the source output will be distorted to the harmonic where the voltage is connected to the nonlinear charge. Since the voltage of the bus bar is harmonic, the linear loads connected to the same bar are also affected by this and draw harmonic current.

Transients are used to describe unwanted events in power systems and they occur in short time interval [5,6]. Transients are sudden increase or decrease in voltage or 
current [7]. Transients occur instantaneously in less time than the half-wave when they are compared with voltage sag and voltage swell. This is the difference between transients and voltage sag, swell. In this short period, voltage amplitude of transients can increase 5-10 times. In other words, transients are changes that occur during the transition from one permanent state to another of a system.

Voltage fluctuation refers to periodic or random variations in the voltage waveform. These changes can the range from $90 \%$ to $110 \%$ of the rated voltage. The flicker is caused by voltage fluctuations and can be recognized by the human eye. It has been determined that the human eye is susceptible to voltage in the $0.5 \mathrm{~Hz}$ to $25 \mathrm{~Hz}$ frequency band. The flicker is caused by voltage fluctuations between 0.5 and $25 \mathrm{~Hz}$ that can be visually perceived in the electric light source.

In this paper, pure sine as a reference, harmonics, flicker and transients are constituted in zero crossing point in MATLAB in order to classify of power quality distortions. The sampling frequency of each signal is 25.6 $\mathrm{kHz}$.

In this paper, feature extraction vector is wanted to obtain. Data size is very important before the classification stage. So data size should be reduced. Most common power quality is dealth with: voltage with harmonics, transient and flicker. Sampling frequency is $25.6 \mathrm{kHz}$. Discrete wavelet transform is applied to these disturbaces. Then energy distribution of voltage with harmonics, transient and flicker is obtained by using Parseval theorem. Energy distribution of these power quality disturbances is compared with the energy distribution of pure sine. Pure sine is selected as reference. It is seen that voltage with harmonics, transient and flicker and pure sine is distinguished by examining their energy distributions. Then amplitude of voltage with harmonics, transient and flicker is increased. Energy coefficients of power quality disturbances and amplitude of power quality disturbances are in direct proportion.

\section{Discrete Wavelet Transform}

Wavelet transform has been proven to very efficient in signal analysis. The wavelet analysis block transforms the distored signal into different time-frequency scales. It is shown in Fig. 1. Wavelet analysis employs the expansion and contraction of basis function to detect simultaneously the characteristics of global and local of measured signal. Wavelets allow the decomposition of a signal into different levels of resolution (frequency octaves). [8] The basis function (Mother Wavelet) is dilated at low frequencies and compressed at high frequencies, so that large windows are used to obtain the low frequency components of the signal, while small windows reflect discontinuities.

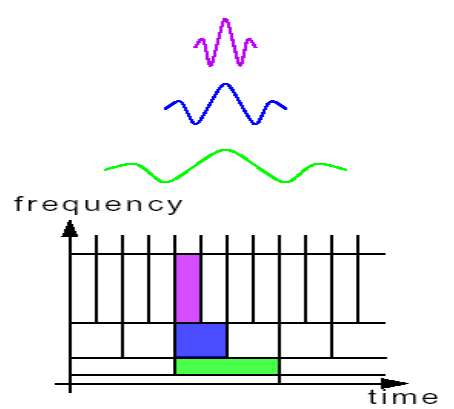

Fig. 1: Time frequency relationship

Daubechies wavelet transform is fundamentally same as Haar wavelet transform, however the only difference is in contents of wavelet and scale function. The following equations describe deriving Daubechies 4 (Daub 4) wavelet and scale functions step by step used in this paper.

Scale vectors for Daub 4 at level 1.

$$
\begin{aligned}
& V_{1}^{1}=\left(\alpha_{1}, \alpha_{2}, \alpha_{3}, \alpha_{4}, 0,0,0, \ldots\right) \\
& V_{2}^{1}=\left(0,0, \alpha_{1}, \alpha_{2}, \alpha_{3}, \alpha_{4}, 0,0,0, \ldots\right) \\
& V_{3}^{1}=\left(0,0,0,0, \alpha_{1}, \alpha_{2}, \alpha_{3}, \alpha_{4}, 0,0,0, \ldots\right) \\
& . . \\
& . . \\
& V_{\frac{N}{2}-1}^{1}=\left(0,0,0, \ldots . \alpha_{1}, \alpha_{2}, \alpha_{3}, \alpha_{4}\right) \\
& V_{\frac{N}{2}}^{1}=\left(\alpha_{1}, \alpha_{2}, 0,0,0, \ldots .0,0, \alpha_{3}, \alpha_{4}\right) \\
& \text { where }
\end{aligned}
$$

and $\mathrm{N}$ is number of samples.

As a generalized expression, Equ. (1) can be re-written as below.

$$
V_{m}^{1}=\alpha_{1} V_{2 m-1}^{0}+\alpha_{2} V_{2 m}^{0}+\alpha_{3} V_{2 m+1}^{0}+\alpha_{4} V_{2 m+2}^{0}
$$

Scale vector for Daub 4 at level 2 is expressed below.

$$
V_{m}^{2}=\alpha_{1} V_{2 m-1}^{1}+\alpha_{2} V_{2 m}^{1}+\alpha_{3} V_{2 m+1}^{1}+\alpha_{4} V_{2 m+2}^{1}
$$

Wavelet vectors for Daub 4 at level 1.

$$
\begin{aligned}
& W_{1}^{1}=\left(\beta_{1}, \beta_{2}, \beta_{3}, \beta_{4}, 0,0,0, \ldots .\right) \\
& W_{2}^{1}=\left(0,0, \beta_{1}, \beta_{2}, \beta_{3}, \beta_{4}, 0,0,0, \ldots\right) \\
& W_{3}^{1}=\left(0,0,0,0, \beta_{1}, \beta_{2}, \beta_{3}, \beta_{4}, 0,0,0, \ldots .\right) \\
& . . \\
& . . \\
& W_{\frac{N}{2}-1}^{1}=\left(0,0,0, \ldots . \beta_{1}, \beta_{2}, \beta_{3}, \beta_{4}\right) \\
& W_{\frac{N}{2}}^{1}=\left(\beta_{1}, \beta_{2}, 0,0,0, \ldots .0,0, \beta_{3}, \beta_{4}\right)
\end{aligned}
$$

where

$$
\beta_{1}=\frac{1-\sqrt{3}}{4 \sqrt{2}}, \beta_{2}=\frac{\sqrt{3}-3}{4 \sqrt{2}}, \beta_{3}=\frac{3+\sqrt{3}}{4 \sqrt{2}}, \beta_{4}=\frac{-1-\sqrt{3}}{4 \sqrt{2}}
$$

and $\mathrm{N}$ is number of samples. 
The relationship between $\beta$ and $\alpha$ is $\beta_{1}=\alpha_{4}$, $\beta_{2}=-\alpha_{3}, \beta_{3}=\alpha_{2}$ and $\beta_{4}=-\alpha_{1}$.

As a generalized expression, Equ. (4) can be re-written as below.

$$
W_{m}^{1}=\beta_{1} V_{2 m-1}^{0}+\beta_{2} V_{2 m}^{0}+\beta_{3} V_{2 m+1}^{0}+\beta_{4} V_{2 m+2}^{0}
$$

Wavelet vector for Daub 4 at level 2 is expressed below.

$$
W_{m}^{2}=\beta_{1} V_{2 m-1}^{1}+\beta_{2} V_{2 m}^{1}+\beta_{3} V_{2 m+1}^{1}+\beta_{4} V_{2 m+2}^{1}
$$

Inverse wavelet transform for Daub 4 is expressed in Equ. (7).

$$
F=A^{1}+D^{1}
$$

In Equ. (7), A is called approximation and D is called detail signal where $\mathrm{F}$ is synthesized signal.

At level 1, A is defined as:

$$
A^{1}=\left(F . V_{1}^{1}\right) V_{1}^{1}+\left(F . V_{2}^{1}\right) V_{2}^{1}+\ldots .+\left(F . V_{N / 2}^{1}\right) V_{N / 2}^{1}
$$

At level 1, D is defined as:

$$
D^{1}=\left(\mathrm{F} \cdot \mathrm{W}_{1}^{1}\right) W_{1}^{1}+\left(\mathrm{F} \cdot \mathrm{W}_{2}^{1}\right) W_{2}^{1}+\ldots .+\left(\mathrm{F} \cdot \mathrm{W}_{\mathrm{N} / 2}^{1}\right) W_{N / 2}^{1}
$$

Inverse wavelet transform for Daub 4 at level 2 is expressed in Equation (10).

$$
F=A^{2}+D^{2}+D^{1}
$$

At level 2, A is defined as:

$$
A^{2}=\left(F \cdot V_{1}^{2}\right) V_{1}^{2}+\ldots .+\left(F \cdot V_{N / 4}^{2}\right) V_{N / 4}^{2}
$$

At level 2, D is defined as:

$$
D^{2}=\left(F \cdot W_{1}^{2}\right) W_{1}^{2}+\ldots .+\left(F \cdot W_{N / 4}^{2}\right) W_{N / 4}^{2}
$$

Fig. (2) shows schematic representation of the mathematical procedure summarized above.

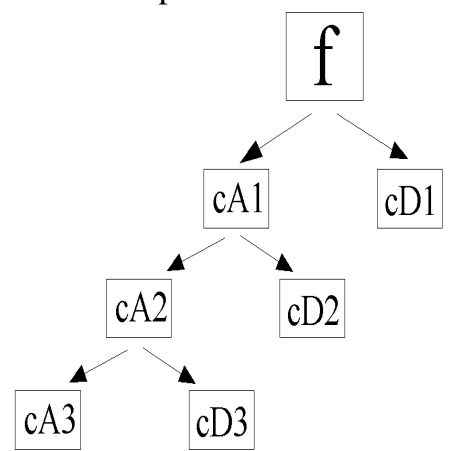

Fig. 2. Signal Decomposition (Mallat Wavelet Tree)

\section{Parseval's Theorem in DWT Application}

In Parseval's theorem, assuming a discrete signal $f[n]$ is the current that flows through the $1 \Omega$ resistance, then the consumptive energy of the resistance is equal to the square sum of the spectrum coefficients of the Fourier transform in the frequency domain [9].

$$
\frac{1}{N} \sum_{n}|f(n)|^{2}=\sum_{k}\left|a_{k}\right|^{2}
$$

where $\mathrm{N}$ is sampling period and $a_{k}$ is the spectrum coefficients of the Fourier transform.

To apply the theorem to the DWT, we use Equ. (13) to obtain equation (14) that is Parseval's theorem in the DWT application [9].

$$
\frac{1}{N} \sum_{t}|f(t)|^{2}=\frac{1}{N_{J}} \sum_{k}\left|a_{J}(k)\right|^{2}+\sum_{J=1}^{J}\left(\frac{1}{N_{J}} \sum_{k}\left|d_{J}(k)\right|^{2}\right)
$$

The first term on the right of Equ.(14) denotes energy of approximation coefficients and the second term on the left of Equ. (14) denotes energy of detail coefficients. The second term giving that energy distribution features of the detail version of distorted signal will be employed to extract the features of power disturbance [9].The process can be represented mathematically by Equation (15).

$$
P_{J}=\frac{1}{N_{J}} \sum_{k}\left|d_{j, k}\right|^{2}=\frac{\left\|d_{J}\right\|^{2}}{N_{J}}
$$

where $\left\|d_{J}\right\|$ is the norm of the expansion coefficient $d_{j}$.

We make the Equation (15) to normalize by Equation (16).

$$
P_{J}^{D}=\left(P_{J}\right)^{1 / 2}
$$

\section{Feauture Extraction Method}

Table I. - Frequency Band Intervals In Multi Resolution Analysis

\begin{tabular}{|c|c|}
\hline $\begin{array}{c}\text { Decomposition } \\
\text { Levels }\end{array}$ & $\begin{array}{c}\text { Frequency } \\
\text { Intervals } \\
\text { (Hz) }\end{array}$ \\
\hline $\mathrm{d} 1$ & $6400-12800$ \\
\hline $\mathrm{d} 2$ & $3200-6400$ \\
\hline $\mathrm{d} 3$ & $1600-3200$ \\
\hline $\mathrm{d} 4$ & $800-1600$ \\
\hline $\mathrm{d} 5$ & $400-800$ \\
\hline $\mathrm{d} 6$ & $200-400$ \\
\hline $\mathrm{d} 7$ & $100-200$ \\
\hline $\mathrm{d} 8$ & $50-100$ \\
\hline $\mathrm{d} 9$ & $25-50$ \\
\hline $\mathrm{d} 10$ & $12.5-25$ \\
\hline $\mathrm{d} 11$ & $6.25-12.5$ \\
\hline $\mathrm{d} 12$ & $3.125-6.25$ \\
\hline $\mathrm{a} 12$ & $0-3.125$ \\
\hline
\end{tabular}


Pure sine, voltage with harmonics, transient and flicker zero crossing point are generated at Matlab for classifying different types of power quality disturbances. Sampling frequeny is $25.6 \mathrm{kHz}$. Daubechies-4 discrete filter is used and 12-level energy distribution coefficients of detail coefficients are obtained by using Equ. 15. d1 and d2 coefficients for transients, $\mathrm{d} 5, \mathrm{~d} 6$ and $\mathrm{d} 7$ for harmonics and $\mathrm{d} 11$ for flicker are important. So, 12 level signal decompomposition are used. Fourier transform (FT) could be used fort his analysis. However FT provides frequency domain but wavelet transform provides both time and frequency domain analysis. The third harmonic with a magnitude of $15 \%$ and the fifth harmonic with a magnitude of $15 \%$ are created along ten periods. $4 \mathrm{kHz}$, $1.5 \mathrm{pu}$ transient are composed at $0.04 \mathrm{sn}$ and 0.1 sn that last $35 \mathrm{~ms}$. Flicker with a magnitude of \%10 that has 58 $\mathrm{Hz}$ inter harmonic is generated along ten periods. Frequency band intervals in multi resolution analysis are given in Table 1. Fig. 3-5 shows energy distribution features of power quality disturbances. Energy distribution features of pure sine is given in Fig. 6 for comparing healthy signal and power quality disturbances. Pure sine is chosen as a reference.

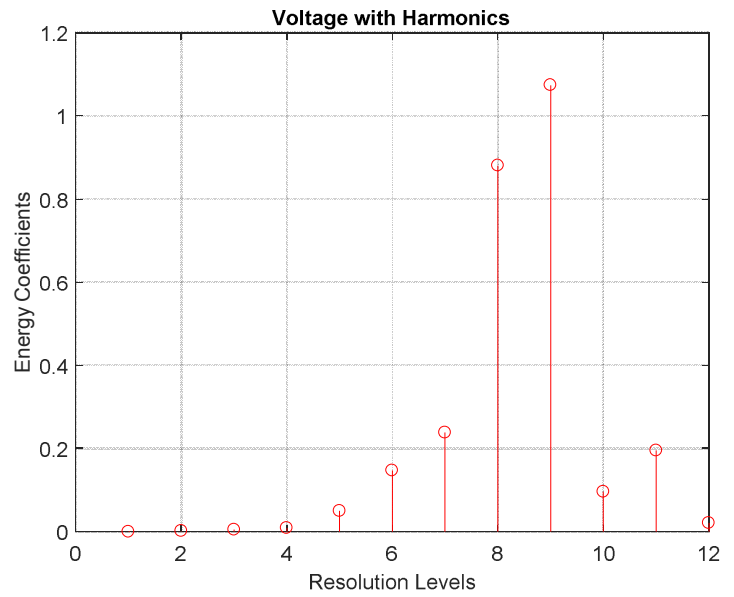

Fig. 3. Energy distribution of voltage with harmonics

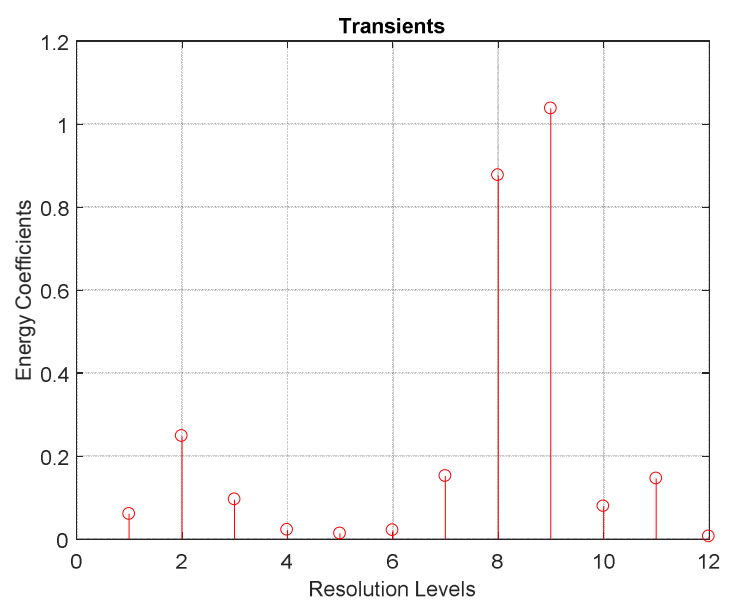

Fig. 4. Energy distribution of voltage with transients

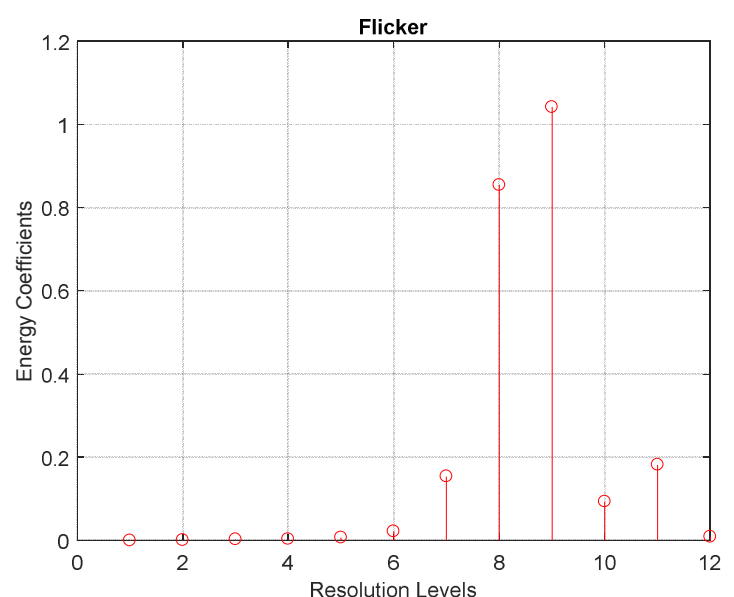

Fig. 5. Energy distribution of flicker

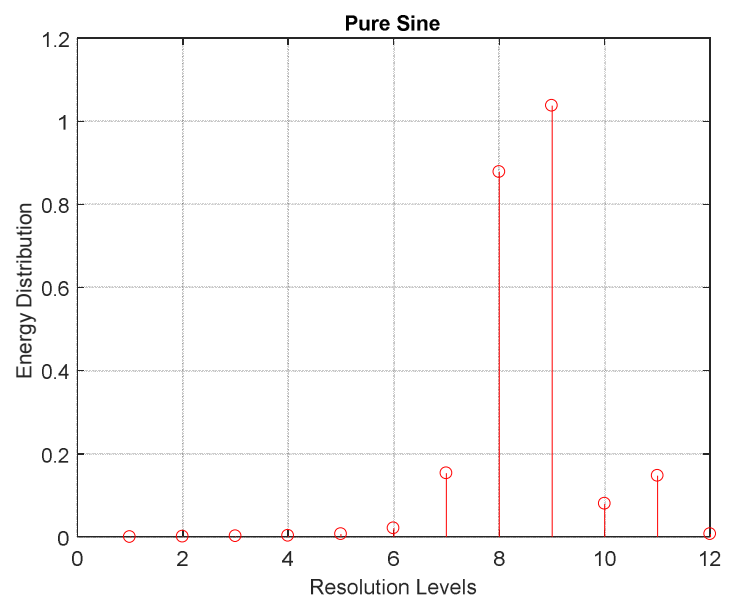

Fig. 6. Energy distribution of pure sine

It has been observed that the related coefficients increase with the frequency interval indicated in Table I when examining energy distribution of detail coefficient of voltage with harmonics, transients, flicker and pure sine. Then the amplitudes of the three PQDs are increased and the method is used again. Distribution of energy coefficients that show distinguishing characteristic of power quality disturbances based on wavelet transform are given in Fig. 7,8,9,10.

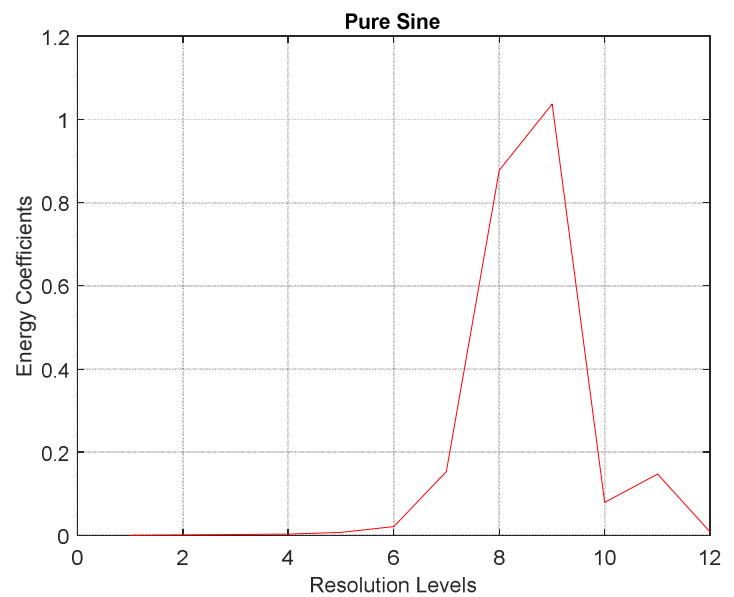

Fig. 7. Distribution of energy coefficients that shows distinguishing characteristic of pure sine based on wavelet transform 


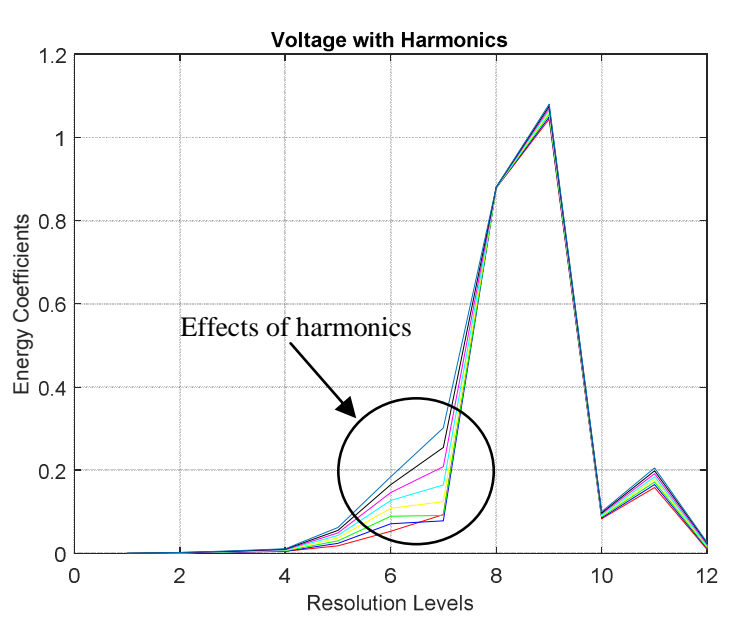

Fig. 8. Distribution of energy coefficients that shows distinguishing characteristic of voltage with harmonics based on wavelet transform

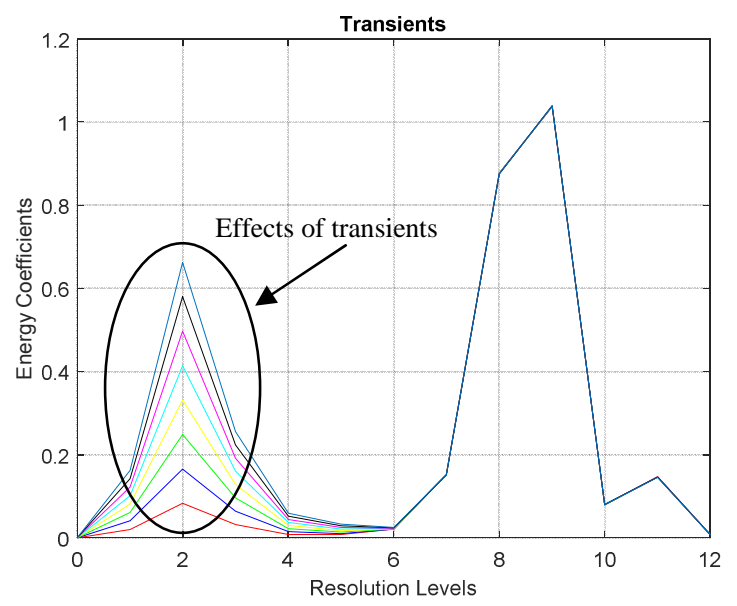

Fig. 9. Distribution of energy coefficients that shows distinguishing characteristic of transients based on wavelet transform

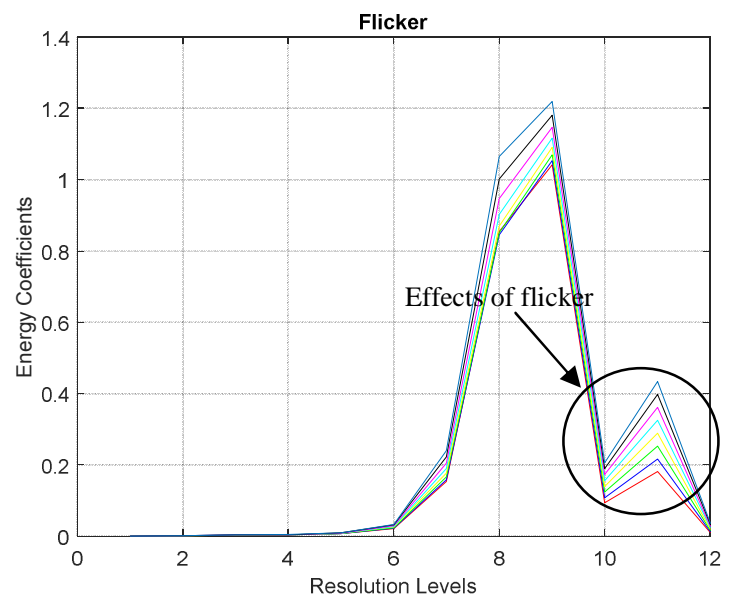

Fig. 10. Distribution of energy coefficients that shows distinguishing characteristic of flicker based on wavelet transform

\section{Conclusion}

In this paper, wavelet based feauture extraction method is applied to power quality disturbances such as voltage with harmonics, transients, flicker and pure sine is used as a reference. Sampling frequency is $25.6 \mathrm{kHz}$ and ten periods of each signal are examined. Signal consisting voltage with harmonics, transients, flicker and pure sine are generated in MATLAB by using mathematical equations. Then feature extraction method based on wavelet transform and Parseval theorem is obtained. The most important stage of classifying is obtaining feauture vector. Thus data size can be reduced. Discrete wavelet transform is applied to pure sine and voltage with harmonics, transients, flicker. Energy distributions of detail coefficients are obtained by decomposing 12 level Daubechies-4 discrete wavelet filter. Equ.16 shows this distribution. Energy distribution of pure sine is obtained as a reference. It is seen that pure sine, voltage with harmonics, transients, flicker can be distinguished visually when looking at the energy distribution of pure sine and these power quality disturbances and data size can be reduced to very small values. When the energy distribution of the voltage with harmonics containing the 3rd and 5th harmonic components is examined, there is a increase in d5, d6 and d7 coefficients. Because there are high frequency components in this voltage signal. Especially according to Table 1, the increase in $\mathrm{d} 6$ and $\mathrm{d} 7$ coefficients is due to the presence of 3rd and 5th harmonic components in the generated signal. In addition, an increase amount is observed at d11 of the 3rd and 5th harmonic signal.The flicker signal with $58 \mathrm{~Hz}$ interharmonics has a flicker frequency of $8 \mathrm{~Hz}$, so increase in d11 coefficient is a distinctive feature. The difference in the energy distributions of the voltage with harmonics and flicker is that the voltage with harmonics have high frequency, so the increase in the $\mathrm{d} 5$, d6 and $\mathrm{d} 7$ in the voltage with harmonics can not be observed in the flicker signal because there is no high frequency in the flicker signal. When energy distribution of $4 \mathrm{kHz}$ transient signal is examined, the significant increase in the coefficients of $\mathrm{d} 1$ and $\mathrm{d} 2$, which express the high frequency component. When we look at the energy distribution features of the generated signals, it is seen that the three different disturbances that we created can be distinguished visually from each other and the data size can be reduced to very low size. Then, the method is analyzed by changing the amplitude of the PQDs. It has been observed that there is a direct proportion between the energy of the wavelet coefficients that define the distortion related to the amplitude of the PQDs. When there is increase in amplitude of PQD, related coefficients rise. This feature makes it easier to classify types of disturbances by methods based on intelligent systems.

Also, this method can be used for real time data. Real time data can be observed by using power quality analyzer and the data can be taken 10 periods length in order to compare analysis results with synthetic analysis results. 


\section{References}

[1] Swain, S. D., Ray, P. K.; Mohanty, K. B., “Improvement of Power Quality Using a Robust Hybrid Series Active Power Filter", IEEE Trans.on Power Elec., 5(32):3490-3498, 2017.

[2] Gaing, Z.L and Huang, H.S., "Wavelet Based Neutral Network for Power Disturbance Classification", IEEE Power Enginerring Soc. Gen. Meet.,Vol. 3: 1621-1628, 2003.

[3] Janik, P., Lobos, T., “Automated Classification of Power Quality Disturbances Using SVM and RBF Networks", IEEE Trans.on Power Elec.,21(3): 1663-1669, 2006.

[4] Hao, L., Feng, Q. W., Qing, S. C., Bin, L. H., “The Multiple Power Quality Disturbance Classification Based on Power System Time Domain Analysis", 2015 Sixth International Conference on Intelligent System Design and Engineering Applications (ISDEA), Vol:4, 867-870.

[5] Atasal, M., 2000, “Güç Kalitesi ve Flicker", Yüksek Lisans Tezi, İstanbul Teknik Üniversitesi Fen Bilimleri Enstitüsü, İstanbul, $146 \mathrm{~s}$.

[6] Pehlivantürk, E., 2004. Güç Kalitesi ve Güç Kalitesi Kontrolörleri. Yüksek Lisans Tezi, Yıldız Teknik Üniversitesi Fen Bilimleri Enstitüsü, İstanbul, $112 \mathrm{~s}$.

[7] IEEEStd 1159-1995. IEEE Recommended Practice for Monitoring Electric Power Quality, IEEE Standards Coordinating Committee 22 on Power Quality, USA.

[8] Kumar, R., Singh, B., Shahani, D. T., Jain, C., “'Dual-Tree Complex Wavelet Transform-Based Control Algorithm for Power Quality Improvement in a Distribution System", IEEE Journals and Magazines, 64(1): 764-772.

[9] Gaing Z.L.,Huang, H.S., “ Wavelet based neural network for power disturbance classification" in 2003 IEEE Power Engineering Society General Meeting, pp. 1621-1628. 